\title{
OPriginal Crmmunications.
}

\section{PATHOIOGICAL AND PRACTICAL RESEARCHES ON THE VARIOUS FORMS OF PARALYSIS.}

By EDWard Meryon, M.D., F.R.C.P. [Continued from page 504 of last volume.]

Paralysis from Effusion and Compression of the Spival CoRd.

THE elaborate mechanism for the transmutation of the impressions of sense into springs of action, which the minute anatomy of the brain and spinal cord reveals to us, acquires a supplementary interest when we come to in. vestigate it as the focus of morbid phenomens. We recognise its importance in the various degrees of arrest which are sometimes observed in its complete development; congenital defects which are seldom, if ever, compatible with the maintenance of life. We see it, too, in one of the earliest, as well as in one of the latest diseases to which human beings are subject; and in both cases paralysis is generally the result.

Hydrorachis, the consequence of a defective develop. ment of the vertebral arches, can appear as a congenital disease only; and although such arrest of genesis may not interfere with intrauterine life and growth, it is in almost all cases fatal shortly after birth. Whether associated with hydrocephalus or not, it generally occa. sions paraplegia, which extends to the sphincters of the rectum and bladder, producing incontinence of fæces and urine.

This is not the place to dwell at length on the anatomy of tumours produced by hydrorachis, beyond the fact that they communicate with the cerebro-spinal fluid which is contained in the vertebral canal; that it is generally between the arachnoid and pia mater, which are not, in their healthy state, in such close apposition in the spine as they are within the skull; and that the paralysing influence is occasioned by the pressure of the fluid on the spinal cord.

On these considerations, various expedients have been suggested for the treatment of spina bifida ; and, in some few instances their practical application has had a suc. cessful result.

Desault recommended a seton to drain off the fluid; but, in every case thus treated, inflammation and constitutional irritation have terminated in death.

Abernethy suggested small and repeated punctures for the same purpose; and two successful cases are recorded by Sir Astley Cooper, together with a graceful homage to the author of the proposition. (Medico.Chi. rurgical Transactions, vol. ii, p. 32.2.)

In one of these cases, the risk of puncturing the tu. mour and setting up constitutional irritation was not incurred, but the disease was treated like a hernia, and the truss was kept applied to prevent the descent of the fluid. At two years of age, the child could walk alone, talked, eventually went to school, and ran, jumped, and played about like other children. He passed well through measles, small-pox, and hooping-cough, and continued to wear the truss, by means of which the fluid was kept entirely within the channel of the spine, but the tumour very soon acquired the size of half a small orange, when the truss was removed. In the second case, Abernethy's suggestion was carried out; the inflammation which ensued produced adhesion of the sides of the sac, which closed the opening from the vertebral canal, and the disease was cured a little more than four months after the first puncture was made.
In neither was there paralysis; and Sir Astley Cooper considered that complication as a reason for not attempting either method of treatment; but Rosetti has reported a case complicated with paraplegia, which was cured, and the motion of the legs restored, by repeated punctures and compression. The palsied state of the sphincter muscles, however, adds immeasurably to the danger and difficulty ; for, of all things it is most important to keep the tumour clean and dry, seeing that under the most favourable circumstances the tense skin is apt to become inflamed.

The treatment of spina bifida by iodine injections. has lately been proposed to the profession; first by Velpeau, and in 1858, $M$. Debout, in a treatise on the The rapeutics of Spina Bifida, gave the details of several cases in which iodine injections have been successfully used. In the following year, Dr. Brainard, of Chicago, recorded the particulars of ten cases, treated by himself and other surgeons, of which four were successful. Again, in 1860, Dr. Gross, of Philadelphia, reported two cases in the North American Medico-Chirurgical Review, one of which terminated successfully. In each case Dr. Gross punctured the tumour about an inch and a quarter from its base, by means of a small flat curved needle which he directed subcutaneously into the sac. Then, through a small cannula he allowed the escape of a drachm of fluid, and injected a solution of one eighth of a grain of iodine, and a quarter of a grain of iodide of potassium. He then closed the puncture by a twisted suture, and coated it over with collodion. Three drops of laudanum were given, and the child was directed to be kept lying on its face. Dr. Gross does not write encouragingly on the operation; still it must be admitted that the general results are favourable. In operating, the puncture should be very small, and the injection very weak at first, the object being to excite a slow process of inflammation in the cyst. It must be confessed, however, that if the life of the child be saved, paraplegia is apt to remain, together with involuntary defæcation and micturition.

Lesions of Sensation and Motion from Congestion of the SpInal Cord.

Spinal congestion is in almost all cases, a result of some specific form of disease; but, as Rokitansky has observed, it also comes on idiopathically. "It then usually pervades the whole cord and its membranes, as well as the brain, and is most frequently met with in early childhood." (Pathological Anatomy. Translated by Moore, vol. iii, p, 448.)

In old people, the veins of the spinal cord which accompany the anterior and posterior spinal arteries, are often found dilated and distended with blood. This, in many instances, may be mere hypostatic engorgement; but cases also occur in which premonitory symptoms lead on to lesions of sensation and motion, which can only be attributed to a congested state of the spinal vessels, and perhaps to effusion into the vertebral canal.

CaSE. A gentleman, aged 76 years, consulted me in May 1849, in consequence of a sense of heaviness and great weakness of the lower extremities, by which he had been inconvenienced for nearly six years, and for which he had taken a brisk purgative every second or third week, with considerable relief. For a month or more, however, the left foot and leg had become somewhat numb and slightly cedematous, and he also experienced so much difficulty and pain in raising and rotating the thigh outward, that be had a room on the ground floor converted into a bedroom. A series of blisters, one every fourth day, over the sacrum and loins, restored very much power to the lower extremities, and enabled him to use the psoas and iliacus muscles without pain. $\mathrm{He}$ had, moreover, a respite from any aggravation of the 
symptoms for some three or four months; still they re. curred, but were always more or less relieved by counterirritants and purgatives until he died of bronchitis, in 1860.*

M. Ollivier relates the case of a locksmith, aged 20 , who, during convalescence from gastric fever, was suddenly seized with paraplegia of the lower extremities. A tingling and pricking sensation extended over the skin from the feet to the epigastrium; and an acute pain extended from the bottom of the back to the sixth dorsal vertebra. On the fourth day, a sloughing sore appeared on the sacrum, and the pain extended to the neck and arms, which were numb and weak. The treatment consisted in the application of blisters; by degrees, the extremities regained their healthy sensation and motion, and in less than a month the patient was discharged cured.

It is perhaps impossible to determine the precise condition of the spinal cord and its vessels which may have occasioned the peculiar symptoms in the two preceding cases. Simple congestion of valveless veins at once sug. gests itself as a cause of the upward course of the morbid action which was manifested in both; but it is improbable that such a state would yield so readily to the influ. ence of a counterirritant, although it certainly does sometimes happen that the progress of an internal disease is arrested by a cutaneous eruption, and the veins of the spine communicate with the superficial veins, either directly or indirectly throughout the whole extent of the vertebral column. Still, the venous sinuses and the veins which accompany the anterior and posterior spinal arteries diminish considerably in the sacral region; and it is scarcely probable that the functional lesion from congestion; would first manifest itself where the veins are smallest.

If, however, the symptoms be attributed to effusion of serum, they admit of a satisfactory interpretation, by the fact that the lowest part of the spinal canal is that to which the fluid naturally gravitates; and there the pia mater, the medium of nutrition to the nervous matter of the cord, would be first exposed to injurious pressure.

Irrespectively, however, of pressure from effusion, there is one circumstance connected with the peculiar mechanism of the vertebral column which is worthy of consideration in cases of spinal congestion, and it is this : that the spinal cord more completely fills the vertebral canal in the dorsal region than it does either above or below, and that, therefore, symptoms of com. pression of the dorsal portion of the cord may present themselves at a very early stage of the disease; but as this fact is, if possible, of still greater importance in redation to myelitis, I will again refer to it.

We may be too ready to attribute morbid phenomena of the nervous system to the influence of pressure from effusion, seeing that in the healthy condition the cerebrospinal fluid serves as a support and protection to the spinal cord and nerves. In old age, too, there is a dis. position in the cord to shrink, and the transudation of serum proceeds pari passu with the diminishing bulk of the organ. So far, therefore, it is a conservative action; but the increased mass of fluid must necessarily produce a sensible effect on the pia mater which surrounds a diminished cord; and it is just in this state of things that we are most likely to obtain relief by the local evacuating $i^{\text {nfluence of blisters. }+}$

* One marked symptom of which the subject of the above case inwariably complained, was the great difficulty he experienced in walking on first rising after a night's rest; and this is generally a feature of spinal congestion.

+ In the treatment of spinal congestion, the ergot of rye has obtained great eelebrity within the last few years in consequence of the property which it eppears to possess of acting on the reso-motor nerves, and thereby diminishing the supply of blo do the cord. To this myelitis.
Paralysis from Meningitis of the Cord.

In meningitis of the spinal cord paralysis does not occur, except from pressure produced by exudation of fluid, or by the extension of inflammation and disorganis. ation of the cord itself.

M. Ollivier has given an interesting case of spontaneous spinal meningitis, in which the symptoms accorded very entirely with the structural lesions. (Traité de la Moelle Epinière, tome ii, p. 551, 2me edition.) The patient, a man, aged 24, was admitted into the Hôtel Dieu with obscure symptoms. On the fifth day of the disease, he had paraplegia, with hyperæsthesia of the lower extremities. Three days afterwards, the arms became partially paralysed, semiflexed, and stiff; the right pupil was more dilated than the left; the association of ideas was slow. Two days subsequently, he died. The cellular tissue which surrounds the dura mater of the spinal canal was filled with vessels injected with blood. The spinal cord was enveloped in a layer of gelatinous yellow matter, between the arachnoid and pia mater. It was most abundant over the lumbar enlargement, and extended as high as the third cervical vertebra. It was thickest where it covered the posterior columns of the cord.

In more tractable cases, after the intensity of the inflammation is subdued by blood-letting and other means calculated to overcome excited action, the paralysing effusion may be drained off by the derivative effect of blisters.

Internal remedies, however, are not to be disregarded; and of these none promise to be more effective than the secale cornutum and the iodide of potassium. The external application of belladonna and chloroform will be found of great service in diminishing the violent pain which accompanies meningitis of the cord. Dr. Ramskill has reported two interesting cases of spinal meningitis, in one of which, paraplegia supervened from rapid effusion of spinal fluid. In both, the iodide of potassium was used, and apparently with good effect; but in the case which manifested no complication of spinal effusion, the ergot of rye was employed in combination with the iodide of potassium. (Medical Times and Gazette, August 31st, 1861.)

Paralysis may result from pressure occasioned by the deposit of cartilaginous or calcareous matter, from consecutive portions of fluid poured out during several mild attacks of spinal meningitis. M. Barbier has recorded a case of paraplegia from such cause. According to Rokitansky (Manual of Pathological Anatomy, vol. iii, p. 440), this deposit is frequently found in the form of scales or laminæ on the inner or visceral surface of the arachnoid, giving rise to what is called ossification of the spinal arachnoid. It seldom happens, however, that the cord is actually compressed by this bony matter; consequently, irritation and affections of a convulsive character are the ordinary forms in which these adventitious growths manifest themselves.

[To be continued.]

Hospital in Constantinople. Letters from Constantinople speak of the great success of the hospital of Faidar Pacha, estabiished there on the French system, and managed by French and Italian professors. The Sultan having recently visited the institution quite unexpectedly, was so delighted with it that he sent for Marco Pacha, Faik Pacha, and Della Suda (an Italian), and, having congratulated them on their management, requested to know what he could do in aid of the hospital. In consequence of their representations, the Sultan has granted a large piece of ground for the erection of a museum and botanical garden, the plants for which are already supplied by the Museum of Natural History in Paris. 